DURNAI, RIS'IT
(Rekayasa Sistem dan Teknologi Informasi)
Vol.5 No. 6 (2021) $1090-1098 \quad$ ISSN Media Elektronik: 2580-0760

\title{
Numerical Approach of Symmetric Traveling Salesman Problem Using Simulated Annealing
}

\author{
Iryanto $^{1}$, P. H. Gunawan ${ }^{2}$ \\ ${ }^{1}$ Informatics Department, Politeknik Negeri Indramayu \\ ${ }^{2}$ School of Computing, Telkom University \\ ${ }^{1}$ iryanto@polindra.ac.id, ${ }^{2}$ phgunawan@ @elkomuniversity.ac.id
}

\begin{abstract}
The aim of this paper is to elaborate the performance of Simulated Annealing (SA) algorithm for solving traveling salesmen problems. In this paper, SA algorithm is modified by using the interaction between outer and inner loop of algorithm. This algorithm produces low standard deviation and fast computational time compared with benchmark algorithms from several research papers. Here SA uses a certain probability as indicator for finding the best and worse solution. Moreover, the strategy of SA as cooling to temperature ratio is still given. Thirteen benchmark cases and thirteen square grid symmetric TSP are used to see the performance of the SA algorithm. It is shown that the SA algorithm has promising results in finding the best solution of the benchmark cases and the squared grid TSP with relative error $0-7.06 \%$ and $0-3.31 \%$, respectively. Further, the SA algorithm also has good performance compared with the well-known metaheuristic algorithms in references.
\end{abstract}

Keywords: simulated annealing, traveling salesman problem, symmetric TSP, square grid TSP.

\section{Introduction}

Finding an optimal route or shortest path is quite challenging due to its own difficulties. The problem can be implemented in some fields. One of them is finding minimum route for a salesman who want to visit place of all of his clients with constraint the places are visited exactly once and the salesman ends his tour in place in which he starts the journey. The problem is well known as TSP (traveling salesman problem) where the problem can be described in weighted graph. Here, the vertex and edges of the graph are used to describe city and distance between two cities, respectively. Moreover, this problem is also known as Hamiltonian circuits problem.

In recent years, many researchers developed and proposed methods to solve the problem. Several methods that has been proposed to solve the problem are discrete tree-seed algorithm (DTSA)[1], black hole algorithm (BHA) [2], discrete lion swarm optimization [3], a hybrid optimization algorithm based on wolf pack search and local search [4], heuristic shortest path algorithm [5], genetic algorithm (GA) [6], particle In this paper, SA algorithm will be elaborated to solve swarm optimization (PSO) [6-8], ant colony the TSP. Moreover, to see the performance of the SA optimization (ACO) [7], shuffled frog leaping algorithm, comparison of SA and well-known algorithms (SFLA) [7], and simulated annealing (SA) metaheuristic algorithm such as BHA, GA, PSO, ACO [9-18]. From [2], BHA has a good performance and state transition algorithm (STA) will be given for

compared with GA, ACO, dan PSO for finding the optimal solution and computational time in 10 benchmark cases of TSP using 22 - 101 number of cities. Meanwhile in [7], ACO known has a good performance compared PSO, improved PSO dan SFLA algorithm for finding the optimal solution of six benchmark cases of TSP with totally $30-100$ cities. However, comparing the computational time, PSO is shown slower than the other algorithms.

Considering the work of Chunhua, et al. in [19], it is shown that STA had the best performance to find the optimal solution and the fastest in computational time for solving three benchmark cases of TSP with the number of cities 16, 48, and 52, compared with ACO and SA. Moreover, STA had low standard deviation from three cases in [19]. This standard deviation is used to show the stability and reliability of algorithm for finding the optimal solution [2]. In [19], each one of algorithms is running in 20 times parallel for solving some problems.

Accepted by Editor: 08-10-2021 | Final Revision: 07-12-2021 | Online Publication: 30-12-2021 
solving some benchmark cases of TSP. Here, SA will be modified by using combination of outer and inner loop in algorithm. In this case, the input of outer loop (the number of experiments) will depend on the result of inner loop (the proses for finding optimal solution). With the modification, it is expected that the performance of the SA can improve.

There are two kinds of TSP namely symmetric and asymmetric TSP [20]. The symmetric TSP is when the distance between city A to city B is the same with the distance between city B to city A. Whereas when the distance is different, the problem is known as Asymmetric TSP. Note that, all mentioned benchmark cases above are belong to the symmetric TSP. The performance comparison will be conducted with three references, [2], [7], and [19]. From these references, there are twelve symmetric TSP benchmark cases where data of each case can be found in [21]. The benchmark cases are Ulysess16, Ulysess22, Bays29, Bayg29, Att48, Ei151, Berlin52, St70, Eil76, Gr96, KroA100, and Eil101. Therefore, the SA algorithm will be focused on solving the symmetric TSP using the benchmark cases to see its performance compared with other algorithms mentioned in those references.

To see further performance of the SA algorithm, another simulation in solving the symmetric TSP cases is conducted. In this case, $n \times n$ cities are well-order generated with distance between two neighboring cities is equal to one. Here, the case is called by name 'square grid symmetric TSP'. The simulations are conducted for $n=3-15$. The values of $n$ are chosen to see performance of the algorithm in solving symmetric TSP cases with small to medium number of cities. Using the values of $n$, numbers of city of the simulations are varied from 9 to 225 .

\section{Research Method}

The simulated annealing (SA) is an optimization method that can be used to solve TSP. The method is used to find shortest route from all possible routes [11]. The optimum solution is obtained when the energy (distance) produced is the minimum and the path tracking is obtained from the route taken. As an optimization method, the difference between SA and other optimization methods is that there is a possibility to still accept worse solution than the current solution to elude local optimal solution [10]. Generally, the SA algorithm is probabilistic metaheuristic method inspired by annealing of metal [11]. The algorithm was introduced in combinatorial problem to solve TSP by Kirkpatrick et al. in 1983 [9]. The idea to accept the worse solution in the process is the main idea that differentiates the algorithm with other optimization methods. The acceptance depends on probability function, $p$, as follows [9]

$$
p(\Delta E, T)=\left\{\begin{array}{cc}
e^{\frac{-\Delta E}{T}} \quad \Delta E>0 \\
1 \quad \Delta E \leq 0
\end{array}\right.
$$

Where $\Delta E$ denotes the energy difference and $T$ represents the temperature.

Note that the energy difference is less or equal than zero, $\Delta E \leq 0$, means that the current solution is better than previous solution. Whereas the difference energy greater than zero, $\Delta E>0$, means that the current solution is worse than previous solution. Criteria for acceptance of the worse solution depends on certain probability, $p r<$ $\frac{-\Delta E}{T}$

$e^{\frac{-\Delta E}{T}}$, where $p r$ is random value between 0 and 1 . In this case, the method utilizes random numbers in the process, so that each experiment, may produce different results and it is still possible that the solution obtained will be trapped in the local optimum solution. Therefore, it takes several trials (running program) to find out the optimum solution.

The cooling process of the temperature is one of factors that need to be considered. At the beginning, the initial value of the temperature is set. The greater the initial temperature used, the wider the range of the random search process [11]. The initial temperature will continue to decrease as the iteration goes to increase. Following [9] and [10], the geometric cooling schedule, $T=T \times r$, is used in here. Value of the cooling coefficient, $r$, is constant between 0 and 1 [9]. Further, value of the coefficient for slow cooling rate is between 0.8 to 0.99 [10]. Therefore, in this article, the cooling rate is $r=0.9$.

In the SA method, at the beginning the initial of solution, $S_{0}$, is given. The solution is randomly generated. Following Algorithm 1 is given the modified SA procedures:

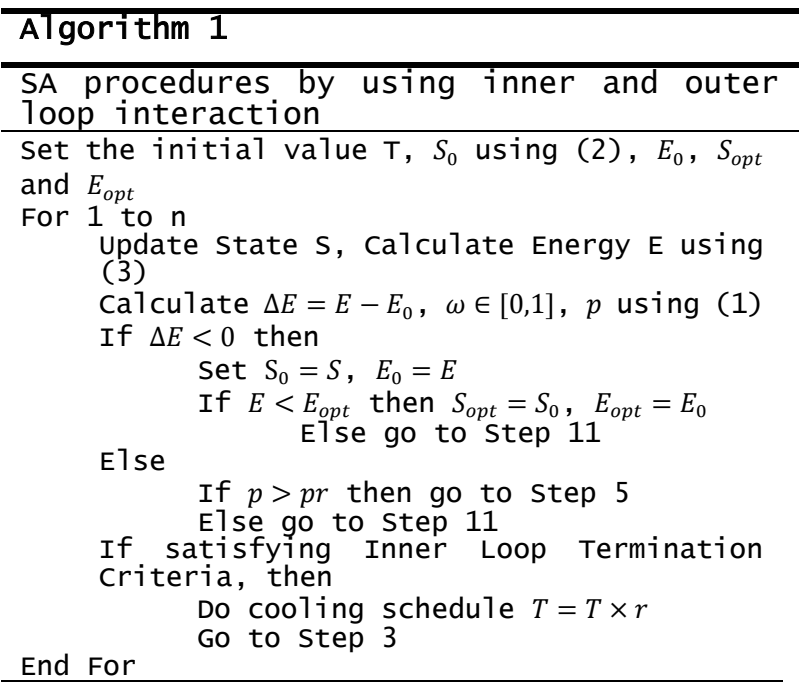

The State (S) is used to track the path taken, for example, there are $\mathrm{M}$ cities to be taken, the state is a number from 1 to $\mathrm{M}$ on the condition that no number is similar. In 
other words, the state is a random permutation of the M 3. Result and Discussion

cities. In this article, the initial state is created as

$$
S_{0}=\{1,2,3,4,5 \ldots, M\}
$$

The state is updated using reversion procedure. In this case, two random values within $\mathrm{M}(\mathrm{k} 1$ and $\mathrm{k} 2)$ are created and position of the state in the range are inversed as described in [16].

Energy (E) is used as an objective function. In this case, it is used to calculate the total distance travelled in one state. Note that in the TSP, the beginning and the end of the route is the same so the additional distance from the last travelled city to the first city is added.

$$
E=\sum_{i=1}^{N-1} d_{i}+d_{N}
$$

Where $d_{i}$ denotes distance between two cities in the state. Position of the cities is expressed in Cartesian coordinates so that the distance can be expresses as given in equation (4).

$$
\begin{aligned}
& d_{i}=\sqrt{\left(S_{x}(i)-S_{x}(i+1)\right)^{2}+\left(S_{y}(i)-S_{y}(i+1)\right)^{2}} \\
& d_{N}=\sqrt{\left(S_{x}(N)-S_{x}(1)\right)^{2}+\left(S_{y}(N)-S_{y}(1)\right)^{2}}
\end{aligned}
$$

Termination of the program can use given maximum iteration or it can use the specified/final temperature. In this article, the stopping criteria is the maximum iteration.
In this paper, two simulations of symmetric TSP are conducted. The first simulation is symmetric TSP based on Data in [21] and the second is square grid symmetric TSP generated in certain ways. In this case $n \times n$ points are well-order generated with distance between two neighboring points is equal to one. Results of the SA algorithm are compared with best known solution (BKS) from references for the symmetric case and analytic solution for the square grid case. Further, comparison with some algorithms is carried out to see the SA algorithm performance. All simulations in this paper are carried out using a PC with windows 10 pro 64-bit (OS), Intel ${ }^{\circledR}$ Core $^{\mathrm{TM}} \mathrm{i} 7-8550 \mathrm{U}$ CPU @ $1.80 \mathrm{GHz}$ processor and 16 GB RAM memory. The SA algorithm is based on $\mathrm{C}++$ language. All simulations are run with parameter $T=1000$ (initial temperature), $r=0.9$, maximum iteration of outer loop is 100 , maximum iteration of inner loop is $800 *$ number of cities.

\subsection{Symmetric TSP}

In this case, all points are generated using TSPLIB data that can be downloaded in [21]. Optimal route of the cases is also available in the reference. The simulations are run for thirteen TSP cases such as Ulysess16, Ulysess22, Bays29, Bayg29, Att48, Eil51, Berlin52, St70, Eil76, Gr96, KroA100, Eil101, and Ch130. The number in the name of the case represents number of cities. Number of cities in the cases are 16, 22, 29, 29, $48,51,52,70,76,96,100,101$, and 130, respectively. These cases are chosen to conduct comparison with some references. Results of the simulations are presented in Table 1.

Table 1. Results of the Symmetric TSP Cases

\begin{tabular}{llllllll}
\hline Case & BS & WS & Ave & IL & Rep & BI & BT (s) \\
\hline Ulysess16 & 73.9876 & 74.4602 & 74.2239 & 12800 & 2 & 593 & 0.333 \\
Ulysess22 & 75.3097 & 76.8119 & 76.2203 & 17600 & 14 & 1334 & 4.349 \\
Bays29 & 9074.15 & 9360.715 & 9202.625 & 23200 & 5 & 622 & 1.239 \\
Bayg29 & 9074.15 & 9576.29 & 9259.601 & 23200 & 38 & 889 & 13.517 \\
Att48 & 33882.48 & 34846.67 & 34766.32 & 38400 & 12 & 38400 & 6.367 \\
Ei151 & 430.89 & 444.74 & 444.41 & 40800 & 52 & 40800 & 31.465 \\
Berlin52 & 7544.366 & 8341.865 & 7845.615 & 41600 & 93 & 41600 & 57.396 \\
St70 & 697.8861 & 727.8492 & 708.807 & 56000 & 31 & 56000 & 32.143 \\
Ei176 & 563.6019 & 594.2049 & 575.5559 & 60800 & 86 & 60800 & 106.068 \\
Gr96 & 539.9611 & 558.2102 & 550.0152 & 76800 & 11 & 76800 & 20.599 \\
KroA100 & 21632.56 & 24181.945 & 22956.741 & 80000 & 17 & 80000 & 32.281 \\
Eil101 & 673.4284 & 708.6054 & 688.9844 & 80800 & 34 & 80800 & 67.967 \\
Ch130 & 6356.304 & 6657.21 & 6443.351 & 104000 & 91 & 104000 & 298.951 \\
\hline
\end{tabular}

In the Table 1, notation BS means the best solution of algorithm, IL is maximum of number iterations of the the SA algorithm, WS is the worst solution of the SA inner loop, Rep means repetition of the outer loop, BI is algorithm, Ave is average of all solution of the SA the best iteration of inner loop to get the best solution,

DOI: https://doi.org/10.29207/resti.v5i6.3549

Creative Commons Attribution 4.0 International License (CC BY 4.0) 
and BT is the total of time (in second) for inner loop and The best solution of the algorithm has the highest and the outer loop to get the best solution. To see performance smallest relative error $7.06 \%$ and $0.0 \%$, respectively. of the SA algorithm, comparison between the results The worst solution has relative error $0.64 \%-13.63 \%$. with the best-known solution (BKS) from references are Whereas average of the solutions has relative error executed. In this case, we calculate relative error using $0.32 \%-9.54 \%$. It is clearly shown in the table that the equation (1) for the best solution, the worst solution, and best performance is in the Ulysess 16 case in which there average of the solution. Further, average time is are 16 points/cities. When the number of points/cities is calculated to see average time for one inner loop using bigger the performance is decreasing, and the average equation (2). The performance of the algorithm is given time is increasing. in Table 2.

It is shown in the Table 2 that results of the SA algorithm are acceptable. According to the relative error, the algorithm has good agreement with the BKS for the conducted cases. The algorithm gets the BKS for Ulysess16, Ulysess22, Bays29, and Bayg29 case. Whereas for the other cases it can be said that the algorithm is also has good comparison with the BKS.

$$
\begin{gathered}
B E=\frac{B S-B K S}{B K S}, W E=\frac{W S-B K S}{B K S}, \\
A E=\frac{A v e-B K S}{B K S} . \\
\text { Average Time }=\frac{B T}{\text { Rep }}
\end{gathered}
$$

Table 2. Performance of the SA algorithm for the Symmetric TSP Cases

\begin{tabular}{lllllll}
\hline Case & BKS & BS & BE & WE & AE & Average Time (s) \\
\hline Ulysess16 & $73.9876[19]$ & 73.9876 & 0.0000 & 0.0064 & 0.0032 & 0.1665 \\
Ulysess22 & $75.3097[2]$ & 75.3097 & 0.0000 & 0.0199 & 0.0121 & 0.3106 \\
Bays29 & $9074.15[8]$ & 9074.15 & 0.0000 & 0.0316 & 0.0142 & 0.2478 \\
Bayg29 & 9074.15 & 9074.15 & 0.0000 & 0.0553 & 0.0204 & 0.3557 \\
Att48 & 33522 & 33882.48 & 0.0108 & 0.0395 & 0.0371 & 0.5306 \\
Ei151 & 426 & 430.89 & 0.0115 & 0.0440 & 0.0432 & 0.6051 \\
Berlin52 & 7542 & 7544.366 & 0.0003 & 0.1061 & 0.0403 & 0.6172 \\
St70 & 675 & 697.8861 & 0.0339 & 0.0783 & 0.0501 & 1.0369 \\
Ei176 & 538 & 563.6019 & 0.0476 & 0.1045 & 0.0698 & 1.2333 \\
Gr96 & $514[22]$ & 539.9611 & 0.0505 & 0.0860 & 0.0701 & 1.8726 \\
KroA100 & 21282 & 21632.56 & 0.0165 & 0.1363 & 0.0787 & 1.8989 \\
Eil101 & 629 & 673.4284 & 0.0706 & 0.1266 & 0.0954 & 1.9990 \\
Ch130 & 6110 & 6356.304 & 0.0403 & 0.0896 & 0.0546 & 3.2852 \\
\hline
\end{tabular}

Comparison is also conducted with other algorithms in Results of the comparison are presented in Table 3. Note several references. The first comparison is with that maximum number of iterations of ACO, PSO, and algorithms in [7]. In the reference, there are six IPSO in the reference for each TSP case is 1000. The algorithms such as ACO, PSO, improved PSO (IPSO), number of ants in ACO is 100 and the number of SFLA, order crossover with inversion mutation (OXIM) populations in PSO and IPSO is equal to number of SFLA, and cycle crossover with inversion mutation cities. Further, it is stated in the reference that in case of (CXIM) SFLA. The algorithm was used to solve six large number of cities, number of ants may be increased symmetric TSP cases namely Oliver30, Ei151, Berlin52, in ACO and number of iterations may be increased in St70, Eil76, and KroA100. All simulations in the IPSO. Thus, the setting of inner loop which is equal to reference are based on MATLAB 6.5 tool and run in PC number of cities times 800 is acceptable. with a $1.70 \mathrm{GHz}$ processor and $4.00 \mathrm{~GB}$ RAM memory. All results given in the reference were gotten after the program was run 1000 times and the time in the reference was an average running time. It is shown in the reference that ACO and Improved PSO have better results according to their error.

Comparisons between the SA algorithm and the other algorithms are carried out for the cases minus Oliver30 case because data of the case are not available in [21].
It is shown in the Table 3 that the SA algorithm has better results than the algorithms in [7] for the TSP cases. The SA algorithm has superior performance than the algorithms in the references. It can be seen also that computational time of the SA algorithm is better than the mentioned algorithm. But the PC specification to run the $\mathrm{SA}$ algorithm is better than the PC specification to run the other algorithm. Note that, value of the average time 
of the SA algorithm is average time for one outer loop 5 independent running are taken and compared with the iteration. SA algorithm and presented in Table 4. To represent the To see further performance of the SA algorithm, another reference setting, the SA algorithm runs with 20000 comparison with other algorithms in [2] and [19] is inner loop iterations and 5 outer loop iterations.

conducted. In this case, there are five compared In [19], performances of SA, ACO, and state transition parameters such as best solution, worst solution, average algorithm (STA) are compared each other to solve of solution, standard deviation, and time. In [2], there are Ulysesss16, Att48, and Berlin52 TSP. All the four algorithms such as ACO, PSO, GA, and BHA that simulations in the reference are based on MATLAB and are compared each other to solve ten symmetric TSP were run on PC with Intel ${ }^{\circledR}$ Core $^{\mathrm{TM}}$ i3-2310M CPU @ cases; Ulysess22, Bays29, Bayg29, Att48, Eil51, 2.10 GHz processor. Note that, in the reference [19], the Berlin52, St70, Eil76, Gr96, and Eil101. All simulations run time is the average time used in 20 execution and the in the reference are based on MATLAB and were maximum iteration for SA is 4000 . Therefore, to adopt executed on PC with Intel ${ }^{\circledR}$ Core ${ }^{\mathrm{TM}} 2$ Duo CPU @ 2 the reference, iterations of inner and outer loop are set $\mathrm{GHz}$ processor and 2 GB RAM memory. Results of the 4000 and 20, respectively. Comparison results are given algorithms using 100 population size, 200 iterations, and in Table 5.

Table 3. Performance Comparison between the SA algorithm and algorithms in [7] for the TSP Cases

\begin{tabular}{|c|c|c|c|c|c|}
\hline Problem & Algorithm & Best & Mean & Error & Average Time \\
\hline \multirow{7}{*}{$\begin{array}{l}\text { Eil51 (51 cities) } \\
\text { BKS }-426\end{array}$} & $\mathrm{ACO}$ & 443 & 516 & 0.0399 & 194 \\
\hline & PSO & 908 & 1313 & 1.1315 & 9 \\
\hline & IPSO & 464 & 543 & 0.0892 & 259 \\
\hline & SFLA & 1169 & 1703 & 1.7441 & 1136 \\
\hline & OXIMSFLA & 534 & 593 & 0.2535 & 16241 \\
\hline & CXIMSFLA & 671 & 671 & 0.5751 & 5147 \\
\hline & $\mathrm{SA}$ & 430.9 & 444.4 & 0.0115 & 0.6051 \\
\hline \multirow{7}{*}{$\begin{array}{l}\text { Berlin52 (52 cities) } \\
\text { BKS - } 7542\end{array}$} & $\mathrm{ACO}$ & 7549 & 9385 & 0.0009 & 276 \\
\hline & PSO & 17296 & 22206 & 1.2933 & 15 \\
\hline & IPSO & 7816 & 8723 & 0.0363 & 469 \\
\hline & SFLA & 19865 & 30598 & 1.6339 & 1150 \\
\hline & OXIMSFLA & 8362 & 9987 & 0.1087 & 21907 \\
\hline & CXIMSFLA & 12266 & 15109 & 0.6264 & 5732 \\
\hline & $\mathrm{SA}$ & 7544.4 & 7845.6 & 0.0003 & 0.6172 \\
\hline \multirow{7}{*}{$\begin{array}{l}\text { St70 (70 cities) } \\
\text { BKS }-675\end{array}$} & $\mathrm{ACO}$ & 707 & 888 & 0.0474 & 1678 \\
\hline & PSO & 2009 & 3411 & 1.9763 & 28 \\
\hline & IPSO & 755 & 871 & 0.1185 & 1058 \\
\hline & SFLA & 2615 & 3759 & 2.8741 & 1859 \\
\hline & OXIMSFLA & 892 & 1004 & 0.3215 & 1771 \\
\hline & CXIMSFLA & 1355 & 1734 & 1.0074 & 3042 \\
\hline & $\mathrm{SA}$ & 697.9 & 708.8 & 0.0339 & 1.0369 \\
\hline \multirow{7}{*}{$\begin{array}{l}\text { eil76 (76 cities) } \\
\text { BKS - } 538\end{array}$} & $\mathrm{ACO}$ & 573 & 659 & 0.0651 & 2035 \\
\hline & PSO & 1662 & 1975 & 2.0892 & 28 \\
\hline & IPSO & 584 & 641 & 0.0855 & 3036 \\
\hline & SFLA & 1904 & 2580 & 2.5390 & 1771 \\
\hline & OXIMSFLA & 733 & 819 & 0.3625 & 99104 \\
\hline & CXIMSFLA & 1072 & 1326 & 0.9926 & 28007 \\
\hline & $\mathrm{SA}$ & 563.6 & 575.6 & 0.0476 & 1.2333 \\
\hline \multirow{4}{*}{$\begin{array}{l}\text { KroA100 (100 } \\
\text { cities) } \\
\text { BKS - } 21282\end{array}$} & $\mathrm{ACO}$ & 22388 & 28655 & 0.0520 & 4516 \\
\hline & PSO & 113174 & 191394 & 4.3178 & 45 \\
\hline & IPSO & 24596 & 28385 & 0.1557 & 9426 \\
\hline & SFLA & 128520 & 175413 & 5.0389 & 3042 \\
\hline
\end{tabular}

DOI: https://doi.org/10.29207/resti.v5i6.3549

Creative Commons Attribution 4.0 International License (CC BY 4.0) 
Iryanto, P. H. Gunawan

Jurnal RESTI (Rekayasa Sistem dan Teknologi Informasi) Vol. 5 No. 6 (2021) 1090 - 1098

Table 3. Performance Comparison between the SA algorithm and algorithms in [7] for the TSP Cases

\begin{tabular}{llllll}
\hline Problem & Algorithm & Best & Mean & Error & Average Time \\
\hline & OXIMSFLA & 37212 & 41371 & 0.7485 & 197264 \\
CXIMSFLA & 58069 & 78451 & 1.7285 & 52365 \\
SA & $\mathbf{2 1 6 3 2 . 6}$ & $\mathbf{2 2 9 5 6 . 7}$ & $\mathbf{0 . 0 1 6 5}$ & $\mathbf{1 . 8 9 8 9}$ \\
\hline
\end{tabular}

Table 4 shows performance comparison of the SA gets the same results with the BHA algorithm. In terms algorithm and the other algorithms in [2]. It is clearly of computational time, it is hard to compare due to the shown in the table that results of the SA algorithm are PC specification but note that the column time for the better than results of the other algorithms in almost every SA algorithm is the total time for running the program case and parameter. The SA algorithm has better with 200 iterations of inner loop and 5 iterations of outer performance in finding the best solution of each case loop.

except for the Ulysess 22 case in which the algorithm

Table 4. Performance Comparison between the SA algorithm and algorithms in [2] for the TSP Cases

\begin{tabular}{|c|c|c|c|c|c|c|}
\hline Problem & Algorithm & Best & Worst & Average & Std. Dev & Time \\
\hline \multirow{5}{*}{$\begin{array}{l}\text { ulysses } 22 \text { (22 cities) } \\
\text { BKS - } 75.3097\end{array}$} & $\mathrm{ACO}$ & 75.3984 & 75.8409 & 75.4869 & 0.19789 & 84.27123 \\
\hline & PSO & 75.9104 & 77.1857 & 76.2186 & 0.55273 & 61.87992 \\
\hline & GA & 75.7744 & 76.4434 & 75.9878 & 1.2307 & 63.39216 \\
\hline & BHA & 75.3097 & 75.9343 & 75.6844 & 0.34208 & 50.44745 \\
\hline & SA & 75.3097 & 76.09488 & 75.5638 & 0.3253 & 1.515 \\
\hline \multirow{5}{*}{$\begin{array}{l}\text { bays } 29 \text { ( } 29 \text { cities }) \\
\text { BKS }-9074.15\end{array}$} & $\mathrm{ACO}$ & 9239.197 & 11014.45 & 9823.202 & 722.4152 & 88.2566 \\
\hline & PSO & 9120.339 & 9498.171 & 9195.905 & 168.9717 & 88.82869 \\
\hline & GA & 9751.426 & 10513.91 & 10015.23 & 319.8788 & 57.11864 \\
\hline & BHA & 9396.475 & 9507.17 & 9463.252 & 60.9588 & 52.1048 \\
\hline & SA & 9076.983 & 9299.9 & 9166.15 & 109.206 & 1.375 \\
\hline \multirow{5}{*}{$\begin{array}{l}\text { bayg29 (29 cities) } \\
\text { BKS - } 9074.15\end{array}$} & $\mathrm{ACO}$ & 9447.493 & 11033.55 & 9882.22 & 675.8331 & 99.95724 \\
\hline & PSO & 9329.251 & 11332.72 & 9947.026 & 799.4073 & 75.29661 \\
\hline & GA & 9579.123 & 10411.2 & 9771.954 & 127.1131 & 56.16117 \\
\hline & BHA & 9375.442 & 9375.442 & 9375.442 & $\mathbf{0}$ & 45.87095 \\
\hline & SA & 9094.635 & 9752.892 & 9314.914 & 278.230 & 1.642 \\
\hline \multirow{5}{*}{$\begin{array}{l}\text { att48 (48 cities) } \\
\text { BKS - 33522 }\end{array}$} & $\mathrm{ACO}$ & 35230.9 & 46204.24 & 39436.18 & 4874.295 & 133.4571 \\
\hline & PSO & 36996.44 & 61421.99 & 47018.41 & 9685.894 & 84.73842 \\
\hline & GA & 35312.52 & 50671.45 & 43620.64 & 2004.001 & 57.35453 \\
\hline & BHA & 34200.86 & 35528.52 & 34473.84 & 589.8024 & 43.21174 \\
\hline & SA & 34056.95 & 34056.95 & 34056.95 & $\mathbf{0}$ & 1.917 \\
\hline \multirow{5}{*}{$\begin{array}{l}\text { eil51 (51 cities) } \\
\text { BKS - } 426\end{array}$} & $\mathrm{ACO}$ & 454.3895 & 469.0531 & 461.0175 & 6.2974 & 59.19328 \\
\hline & PSO & 469.1551 & 737.5258 & 574.8022 & 107.2371 & 57.25646 \\
\hline & GA & 448.8397 & 462.1142 & 453.4773 & 9.4157 & 59.63916 \\
\hline & BHA & 437.893 & 526.8977 & 458.9252 & 38.6365 & 44.39009 \\
\hline & SA & 429.484 & 476.9822 & 446.996 & 17.4654 & 2.568 \\
\hline \multirow{5}{*}{$\begin{array}{l}\text { berlin52 (52 cities) } \\
\text { BKS - } 7542\end{array}$} & $\mathrm{ACO}$ & 7757.026 & 10541.12 & 8522.902 & 1152.2 & 65.07013 \\
\hline & PSO & 9218.468 & 14279.43 & 11089.53 & 2067.932 & 68.64806 \\
\hline & GA & 8779.756 & 9565.374 & 9288.448 & 1301.211 & 52.73534 \\
\hline & BHA & 8188.071 & 9356.748 & 8455.83 & 508.9871 & 43.40446 \\
\hline & SA & 7957.667 & 8074.314 & 7997.202 & 49.713 & 1.907 \\
\hline \multirow{5}{*}{$\begin{array}{l}\text { st70 (70 cities) } \\
\text { BKS }-675\end{array}$} & $\mathrm{ACO}$ & 711.6515 & 855.2032 & 757.754 & 59.6079 & 94.56822 \\
\hline & PSO & 1030.848 & 1756.123 & 1321.814 & 269.2793 & 55.28412 \\
\hline & GA & 1112.308 & 1242.201 & 1158.846 & 52.1734 & 55.09585 \\
\hline & BHA & 723.2691 & 1081.109 & 797.5745 & 125.2272 & 45.3308 \\
\hline & SA & 702.9316 & 705.1066 & 703.802 & 1.066 & 2.312 \\
\hline \multirow{5}{*}{$\begin{array}{l}\text { eil76 (76 cities) } \\
\text { BKS - } 538\end{array}$} & $\mathrm{ACO}$ & 574.2404 & 665.9995 & 594.1442 & 40.2152 & 61.7418 \\
\hline & PSO & 804.2667 & 1195.902 & 975.6397 & 152.4061 & 56.76708 \\
\hline & GA & 619.2262 & 679.7864 & 652.0593 & 122.0972 & 46.69151 \\
\hline & BHA & 566.243 & 925.8417 & 659.1021 & 152.1754 & 46.54038 \\
\hline & SA & 564.345 & 595.6808 & 582.495 & 11.924 & 2.499 \\
\hline \multirow{3}{*}{$\begin{array}{l}\text { gr96 (96 cities) } \\
\text { BKS }-514\end{array}$} & $\mathrm{ACO}$ & 555.7535 & 639.9167 & 580.5406 & 33.9301 & 84.38977 \\
\hline & PSO & 1095.111 & 1728.824 & 1378.87 & 247.5099 & 56.21171 \\
\hline & GA & 737.9671 & 748.3543 & 742.4275 & 4.3282 & 63.24444 \\
\hline
\end{tabular}

DOI: https://doi.org/10.29207/resti.v5i6.3549

Creative Commons Attribution 4.0 International License (CC BY 4.0) 
Iryanto, P. H. Gunawan

Jurnal RESTI (Rekayasa Sistem dan Teknologi Informasi) Vol. 5 No. 6 (2021) 1090 - 1098

Table 4. Performance Comparison between the SA algorithm and algorithms in [2] for the TSP Cases

\begin{tabular}{lllllll}
\hline Problem & Algorithm & Best & Worst & Average & Std. Dev & Time \\
\hline & BHA & 546.8397 & 1197.876 & 807.2465 & 258.815 & 43.58791 \\
eil101 (101 cities) & SA & $\mathbf{5 3 9 . 4 2 6 8}$ & $\mathbf{5 3 9 . 4 2 6 8}$ & $\mathbf{5 3 9 . 4 2 6 8}$ & $\mathbf{0}$ & $\mathbf{2 . 9 6 9}$ \\
BKS -629 & ACO & 725.0996 & 868.2047 & 763.9207 & 59.9684 & 89.63974 \\
& PSO & 1158.704 & 1973.819 & 1499.991 & 319.7468 & 62.09302 \\
& GA & 828.8806 & 854.4381 & 838.8307 & 9.9642 & 55.18821 \\
& BHA & 720.3838 & 1249.868 & 897.3813 & 210.1446 & 45.83337 \\
& SA & $\mathbf{6 8 7 . 9 9 4 9}$ & $\mathbf{7 0 4 . 1 8 1 9}$ & $\mathbf{6 9 7 . 7 0 7}$ & $\mathbf{7 . 9 3 0}$ & $\mathbf{3 . 1 6 6}$ \\
\hline
\end{tabular}

Performance of the SA algorithm compared with algorithm is better than the other algorithms in algorithms in [19] is shown in Table 5. It is clearly remaining parameters. Neglected the STA, the SA shown that performance the SA algorithm is in good algorithm has the better performance compared with comparison with the other algorithms. In terms of the ACO given in [19]. Note that, value of the average time best solution, the SA algorithm has the same result with of the SA algorithm is average time for one outer loop STA in Ulysess 16 and Berlin52 TSP and better result in iteration.

Att48 TSP case. Further, in Ulysess16 TSP case, the SA

Table 5. Performance Comparison between the SA algorithm and algorithms in [19] for the TSP Cases

\begin{tabular}{lllllll}
\hline Problem & Algorithm & Best & Worst & Average & Std. Dev & Average Time \\
\hline ulysess16 (16 cities) & ACO & 74.6287 & 78.7728 & 76.0864 & 1.1062 & 11.3038 \\
BKS -73.9876 & STA & 73.9876 & 74.5939 & 74.0779 & 0.1626 & 1.2223 \\
& SA & $\mathbf{7 3 . 9 8 7 6}$ & $\mathbf{7 3 . 9 9 9 8}$ & $\mathbf{7 3 . 9 9 3 7}$ & $\mathbf{0 . 0 0 6 1}$ & $\mathbf{0 . 2 5 9 3}$ \\
att48 (48 cities) & ACO & 37015 & 39801 & 38449 & 862.4546 & 102.4784 \\
BKS -33522 & STA & 33724 & 36205 & 34872 & 668.7553 & 3.0462 \\
& SA & $\mathbf{3 3 7 1 0 . 9 9}$ & 36568.22 & 34946.77 & 777.57 & $\mathbf{0 . 2 4 4 2}$ \\
berlin52 (52 cities) & ACO & 8240.4 & 9151.3 & 8777.6 & 267.1124 & 118.0948 \\
BKS -7542 & STA & 7544.4 & $\mathbf{8 6 3 0 . 5}$ & 8247.2 & 273.4509 & 3.3438 \\
& SA & $\mathbf{7 5 4 4 . 4}$ & 8918.8 & $\mathbf{7 7 0 6 . 5}$ & 360.1 & $\mathbf{0 . 2 3 6 9}$
\end{tabular}

In the SA algorithm, random value is used, therefore its cities. Using the values of $n$, numbers of city of the result is influenced by the value. Depends on the value, simulations are varied from 9 to 225 . Results of the the algorithm may get better result in faster simulations are presented in Table 6 and Table 7. The computational time or it is possible to get worse solution best solution of the case for $n=7$ can be seen in Figure since SA algorithm has possibility to accept worse 1. solution leading to get local optimum. Repetitions of the program execution are to see the convergence of the results. The lower standard deviation shows that the related algorithm is more stable and reliable in finding the optimal/best solution [2].

\subsection{Square Grid Symmetric TSP}

In the square grid symmetric TSP, $n \times n$ cities are wellorder generated with distance between two neighboring cities is equal to one. In this case, simulations are conducted for $n=3-15$. The values of $n$ are chosen to see performance of the algorithm in solving symmetric TSP cases with small to medium number of

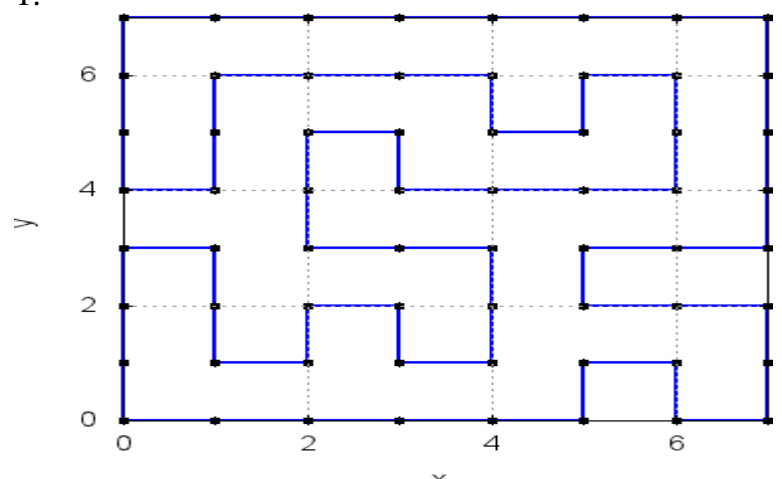

Figure 1. Solution of Square Grid Symmetric TSP for $n=7$

\begin{tabular}{|c|c|c|c|c|c|c|c|c|}
\hline $\mathrm{n}$ & $\begin{array}{l}\text { Number } \\
\text { of City }\end{array}$ & $\mathrm{BS}$ & WS & Ave & IL & Rep & $\mathrm{BI}$ & BT \\
\hline 3 & 9 & 9.4142 & & & 7200 & 0 & 154 & \\
\hline 4 & 16 & 16 & & & 12800 & 0 & 445 & \\
\hline 5 & 25 & 25.4142 & & & 20000 & 0 & 1353 & \\
\hline 6 & 36 & 36 & 36.8284 & 36.7249 & 28800 & 8 & 12353 & 2.841 \\
\hline
\end{tabular}

DOI: https://doi.org/10.29207/resti.v5i6.3549

Creative Commons Attribution 4.0 International License (CC BY 4.0) 
Iryanto, P. H. Gunawan

Jurnal RESTI (Rekayasa Sistem dan Teknologi Informasi) Vol. 5 No. 6 (2021) 1090 - 1098

\begin{tabular}{|c|c|c|c|c|c|c|c|c|}
\hline 7 & 49 & 49.4142 & 51.0711 & 50.5188 & 39200 & 3 & 21047 & 1.216 \\
\hline 8 & 64 & 64 & 66.4853 & 64.9527 & 51200 & 60 & 36647 & 54.219 \\
\hline 9 & 81 & 81.4142 & 86.3848 & 82.57401 & 64800 & 65 & 57198 & 83.574 \\
\hline 10 & 100 & 100.8284 & 104.1421 & 103.0376 & 80000 & 3 & 80000 & 4.377 \\
\hline 11 & 121 & 123.0711 & 125.5563 & 124.5622 & 96800 & 5 & 96800 & 10.964 \\
\hline 12 & 144 & 147.3137 & 152.2843 & 150.9036 & 115200 & 12 & 115200 & 43.283 \\
\hline 13 & 169 & 174.3848 & 177.6985 & 176.5939 & 135200 & 3 & 135200 & 12.852 \\
\hline 14 & 196 & 201.799 & 208.4264 & 206.2173 & 156800 & 3 & 156800 & 16.894 \\
\hline 15 & 225 & 232.8701 & 237.8406 & 235.7136 & 180000 & 37 & 180000 & 330.465 \\
\hline
\end{tabular}

Table 7. Performance of the SA algorithm for the Square Grid TSP Cases

\begin{tabular}{|c|c|c|c|c|c|}
\hline $\mathrm{n}$ & Number of City & BKS & $\mathrm{BS}$ & Error & Average Time \\
\hline 3 & 9 & 9.4142 & 9.4142 & $\mathbf{0}$ & \\
\hline 4 & 16 & 16 & 16 & $\mathbf{0}$ & \\
\hline 5 & 25 & 25.4142 & 25.4142 & $\mathbf{0}$ & \\
\hline 6 & 36 & 36 & 36 & $\mathbf{0}$ & 0.3551 \\
\hline 7 & 49 & 49.4142 & 49.4142 & $\mathbf{0}$ & 0.4053 \\
\hline 8 & 64 & 64 & 64 & $\mathbf{0}$ & 0.9037 \\
\hline 9 & 81 & 81.4142 & 81.4142 & $\mathbf{0}$ & 1.2858 \\
\hline 10 & 100 & 100 & 100.8284 & 0.0083 & 1.4590 \\
\hline 11 & 121 & 121.4142 & 123.0711 & 0.0136 & 2.1928 \\
\hline 12 & 144 & 144 & 147.3137 & 0.0230 & 3.6069 \\
\hline 13 & 169 & 169.4142 & 174.3848 & 0.0293 & 4.284 \\
\hline 14 & 196 & 196 & 201.799 & 0.0296 & 5.6313 \\
\hline 15 & 225 & 225.4142 & 232.8701 & 0.0331 & 8.9315 \\
\hline
\end{tabular}

It is shown in Table 7 that performance of the SA SA algorithm by parallel algorithm can be considered for algorithm are acceptable. The algorithm has no error in the future work.

finding the optimal solution for cases with number of cities are 9, 16, 25, 49, 84, and 81. For the remaining Acknowledgements cases, the error is getting larger when the number of the cities are getting bigger. The error are $0 . \%-3.31 \%$ which mean the performance of the SA algorithm is good.

\section{Conclusion}

Elaboration of simulated annealing algorithm using inner and outer loop has been carried out. Several simulations have been conducted to see performance of the algorithm. The simulations are carried out for 13 symmetric TSP cases taken from [21]. The algorithm shows promising performance with relative error; the best solution $0-7.06 \%$, the worst solution $0.64 \%$ $13.63 \%$, and average of the solutions $0.32 \%-9.54 \%$. The algorithm also has good performance compared with the well-known metaheuristic algorithms. The SA algorithm has better performance in finding the best solution compared with the other algorithms in [2], [7], and [19]. Moreover, the SA algorithm has small enough standard deviation value $0-777.57$ with average value 124.118. In the square grid TSP, the SA algorithm also has good performance with relative error $0-3.31 \%$. The SA algorithm can find the best solution of the cases. However, in some cases the SA could not find the best solution. Here, existence of the random value influences result of the algorithm. For future work, adding local search algorithm is expected to increase the performance of SA. Moreover, decreasing the computational time of

Authors want to say thank you to Telkom University for research funding.

\section{References}

[1] A. C. Cinar, S. Korkmaz, and M. S. Kiran. "A discrete tree-seed algorithm for solving symmetric traveling salesman problem." Engineering Science and Technology, an International Journal, vol. 23, no. 4, pp. 879-890, 2020.

[2] A. Hatamlou. "Solving travelling salesman problem using black hole algorithm." Soft Computing, vol. 22, no. 24, pp. 8167-8175, 2018.

[3] Z. Daoqing and J. Mingyan. "Parallel discrete lion swarm optimization algorithm for solving traveling salesman problem." Journal of Systems Engineering and Electronics, vol. 31, no. 4 pp. 751-760, 2020.

[4] R. Dong, S. Wang, G. Wang, and X. Wang. "Hybrid optimization algorithm based on wolf pack search and local search for solving traveling salesman problem." Journal of Shanghai Jiaotong University (Science), vol. 24, no. 1, pp. 41-47, 2019.

5] S. A. Bakar and M. Ibrahim. "Optimal solution for travelling salesman problem using heuristic shortest path algorithm with imprecise arc length." In AIP Conference Proceedings, AIP Publishing LLC, vol. 1870, no. 1, pp. 040061, 2017.

[6] Panda, Madhumita. "Performance Comparison of Genetic Algorithm, Particle Swarm Optimization and Simulated Annealing Applied to TSP." International Journal of Applied Engineering Research, vol. 13, no. 9, pp. 6808-6816, 2018.

[7] S. Saud, H. Kodaz, and I. Babaoğlu, "Solving Travelling Salesman Problem by Using Optimization Algorithms" in The 9th International Conference on Advances in Information Technology, KnE Life Sciences, pp 17-32, 2017.

[8] X. Wang, A. Mu, and S. Zhu. "ISPO: A new way to solve traveling salesman problem." Intelligent Control and Automation, vol. 4, no. $2,2013$. 
[9] Zhou, Ai-Hua, Li-Peng Zhu, Bin Hu, Song Deng, Yan Song, Hongbin Qiu, and Sen Pan. "Traveling-salesman-problem algorithm based on simulated annealing and gene-expression programming." Information, vol. 10, no. 1, 2019.

[10]A. E. S. Ezugwu, A. O. Adewumi, and M. E. Frîncu. "Simulated annealing based symbiotic organisms search optimization algorithm for traveling salesman problem." Expert Systems with Applications, vol. 77, pp. 189-210, 2017.

[11]Zhan, Shi-hua, Juan Lin, Ze-jun Zhang, and Yi-wen Zhong. "Listbased simulated annealing algorithm for traveling salesman problem." Computational intelligence and neuroscience, 2016.

[12]A. Khumaidi, R. Raafi'udin, and I. P. Solihin. (2020) "Simulation Of Traveling Salesman Problem For Distribution Of Fruits In Bogor City With Simulated Annealing Method.” Jurnal Mantik, vol. 3, no. 4, pp. 611-618, 2020.

[13]C. Wang, M. Lin, Y. Zhong, and H. Zhang. "Swarm simulated annealing algorithm with knowledge-based sampling for travelling salesman problem." International Journal of Intelligent Systems Technologies and Applications, vol. 15, no. 1, pp. 74-94, 2016.

[14]M. Makuchowski. "Effective algorithm of simulated annealing for the symmetric traveling salesman problem." In International Conference on Dependability and Complex Systems (pp. 348359). Springer, Cham, July. 2018.

[15]X. Han, Y. Dong, L. Yue, and Q. Xu. "State transition simulated annealing algorithm for discrete-continuous optimization problems." IEEE Access, 7, 44391-44403, 2019.
[16]L. Xiong and S. Li. "Solving TSP Based on the Improved Simulated Annealing Algorithm with Sequential Access Restrictions." In 2016 6th International Conference on Mechatronics, Computer and Education Informationization (MCEI 2016), Atlantis Press, pp. 610-616, 2016.

17]M. Rahbari and A. Jahed. "A Hybrid Simulated Annealing Algorithm for Travelling Salesman Problem with Three Neighbor Generation Structures". In 10th International Conference of Iranian Operations Research Society (ICIORS 2017), Babolsar, Iran, May. 2017.

[18]L. Wang, R. Cai, M. Lin, and Y. Zhong. "Enhanced list-based simulated annealing algorithm for large-scale traveling salesman problem." IEEE Access, 7, 144366-144380, 2019.

[19] Y. Chunhua, T. Xiaolin, Z. Xiaojun, and G. Weihua. "State transition algorithm for traveling salesman problem." In Proceedings of the 31st Chinese Control Conference, IEEE, pp. 2481-2485, 2012

20]E. Osaba, J. Del Ser, A. Sadollah, M. N. Bilbao, and D. Camacho. "A discrete water cycle algorithm for solving the symmetric and asymmetric traveling salesman problem." Applied Soft Computing, 71, 277-290, 2018.

[21]G. Reinelt, “TSPLIB", 19 February 1997. Available: http://elib.zib.de/pub/mp-testdata/tsp/tsplib/tsplib.html [Accessed on 18 January 2021]

22]P. H. Siqueira, S. Scheer, and M. T. A. Steiner. "A recurrent neural network to traveling salesman problem." Travelling Salesman Problem, I-Tech Veinna, pp. 135-156, 2008. 